\title{
INTEGRATION OF A LOW-COST GLOBAL NAVIGATION SATELLITE SYSTEM TO A SINGLE-BOARD COMPUTER USING KALMAN FILTERING
}

\author{
Thales M. de A. Silva ${ }^{1^{*}}$, Grégory de O. Mayrink², Domingos S. M. Valente², Daniel M. Queiroz ${ }^{2}$ \\ ${ }^{1 *}$ Corresponding author. Universidade Federal de Viçosa/ Viçosa - MG, Brasil. \\ E-mail: thalesmaurino@gmail.com | ORCID ID: http://orcid.org/0000-0002-1924-6671
}

\section{KEYWORDS}

georeferencing, positioning, beaglebone black, algorithm.

\begin{abstract}
The global navigation satellite system (GNSS) is the basis for localized crop management by allowing the georeferencing of collected data and the generation of maps by different systems that compose precision agriculture. There is a demand for low-cost navigation systems to enable their use in agriculture. Therefore, the objective of this study is to integrate a low-cost GNSS module to a single-board computer using Kalman filtering to obtain navigation data. The system was evaluated by performing one static and two kinematic experiments, with three repetitions each. In the static experiment, the mean error was $3.25 \mathrm{~m}$ with a root mean square error (RMSE) of $3.73 \mathrm{~m}$. In the first kinematic experiment, data variability was lower at a velocity of $1.39 \mathrm{~m} \mathrm{~s}^{-1}$. In the second kinematic experiment, the mean error was 1.26 and $1.13 \mathrm{~m}$, and the RMSE was 1.45 and $1.27 \mathrm{~m}$ for data obtained before and after filtering, respectively. In conclusion, the system reduces the lateral errors in linear sections but is not indicated for sections that change direction. Moreover, this system can be used in agricultural applications such as soil sampling and crop yield monitoring.
\end{abstract}

\section{INTRODUCTION}

Global navigation satellite systems (GNSS) are one of the pillars of precision agriculture (PA) (De Oliveira, 2016). GNSS are the basis for localized crop management and are used during the stages of planting, agrochemical application, and harvesting (Chen et al., 2005; Suprem et al., 2013). In addition, GNSS allow the georeferencing of the collected data and the generation of maps by the different systems that compose PA. The localized management of agricultural processes distinguishes PA from conventional agriculture (Mondal et al., 2011).

More recently, there has been a lot of interest in ground-based robotic vehicles and remotely-piloted aircraft in PA (Bakker et al., 2011; Ji et al., 2012; Mousazadeh, 2013; Yin \& Noguchi, 2013). However, the cost of navigation systems needs to be reduced to make robotic systems more feasible, especially in small farms (De Oliveira, 2016). Therefore, technologies that facilitate access to GNSS are fundamental to enable the navigation of small agricultural machines. Single-board computers, such as BeagleBoard Black (BBB; model Revision C, Michigan, USA) are low-cost computers that allow the rapid development of new tools for PA (Olesen et al., 2016).
Another critical factor to consider when implementing a GNSS in agricultural machinery is positioning accuracy. Precision is determined primarily by the measurement noise (uncertainty) and the type of navigation algorithm (Laveti et al., 2016). In practice, the measurement uncertainty never reaches zero even if the parameters and noise in the system are effectively modeled (Xu \& Xu, 2016).

The navigation algorithm should make an optimal estimate of these uncertainties to remove them from the signal and consequently improve accuracy (Fourati, 2015; Li et al., 2017). The use of Kalman filtering (Kalman, 1960) is one strategy to remove signal errors in low-cost navigation systems (Huang et al., 2018; Xiong et al., 2018; Liu et al., 2018). The objective of this study is to evaluate the accuracy of an integrated system composed of a lowcost GNSS module, a BBB single-board computer, and Kalman filtering to obtain navigation data.

\section{MATERIAL AND METHODS}

A Revision C.1 BBB platform with a Linux Debian operating system version 7.8 (Wheezy) (USD 63.00) was used. A Simply Tronics GNSS receiver module model VPN1513 was integrated into this platform. This integration

\footnotetext{
${ }^{2}$ Universidade Federal de Viçosa/ Viçosa - MG, Brasil.

Received in: 4-17-2018

Accepted in: 3-19-2019
} 
allowed implementing a filtering algorithm in the positioning data.

The adopted GNSS module is low-cost (USD 20.00), uses the SiRF Star III chipset with 20 satellite reception channels, has a sensitivity of $-159 \mathrm{dBm}$ and precision of $+/-$ $10 \mathrm{~m}$, and captures signals at a frequency of $1575.42 \mathrm{MHz}$ (band L1). The module was powered by the BBB with a voltage of $5 \mathrm{~V}$. The BBB was powered with $5 \mathrm{~V}$ via a USB cable using an external battery commonly used to recharge mobile phones (USD 10.00).

The connection between the GNSS module and the BBB platform was performed via Universal Asynchronous Receiver/Transmitter (UART) serial ports. The chosen UART serial ports were UART1_TXD and UART1_RXD, located in the BBB expansion header P9 with 24 and 26 pins, respectively. Serial communication was performed at a transmission rate of 9600 because this speed was sufficient to receive all data. The data were updated every second until the user terminated the communication.

\section{Implementation of the filtering algorithm}

An algorithm was developed using the $\mathrm{C}++$ language to communicate with the GNSS module (Figure 1). The algorithm received data from the GNSS module in the format NMEA 0183, which is the GNSS communication protocol (Khan \& Mishra, 2012). The algorithm converted the data to latitude and longitude in WGS 1984 using decimal degrees and later to the metric projection UTM WGS 1984. This last transformation was performed using a protocol developed by Dutch (2015) adapted to the $\mathrm{C}++$ language.

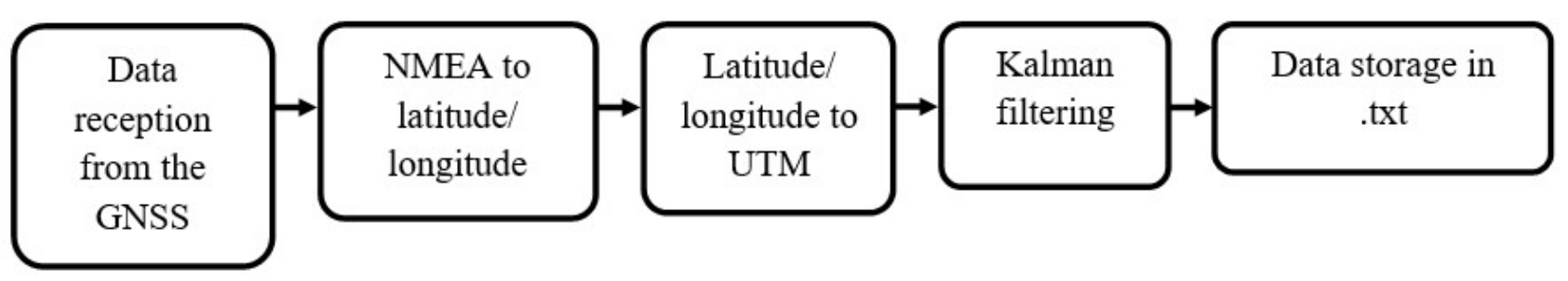

FIGURE 1. Stages of the filtering algorithm in $\mathrm{C}++$.

The accuracy of positioning data provided by the GNSS module was improved by implementing a navigation algorithm in $\mathrm{C}++$ language based on Kalman filtering. The Kalman filter has predictive and corrective characteristics and operates using the least squares method (Cintra et al., 2010). Kalman filtering is a recursive approach involving ideal linear filtering to estimate the state variables of a system. For system modeling, the initial positioning values were used to predict and adjust parameters in each new measurement, obtaining the estimated error in each data update (Shen et al., 2015).

Kalman filtering included two main steps, prediction and correction (data update), which were subdivided into five steps (Figure 2). The first two steps involved prediction. In this step, an apriori estimate of each sample point was made on the basis of the current status and the system model. After that, an improved a posteriori estimate was designed on the basis of the last three steps, therefore completing the update step as described by Li et al. (2016).

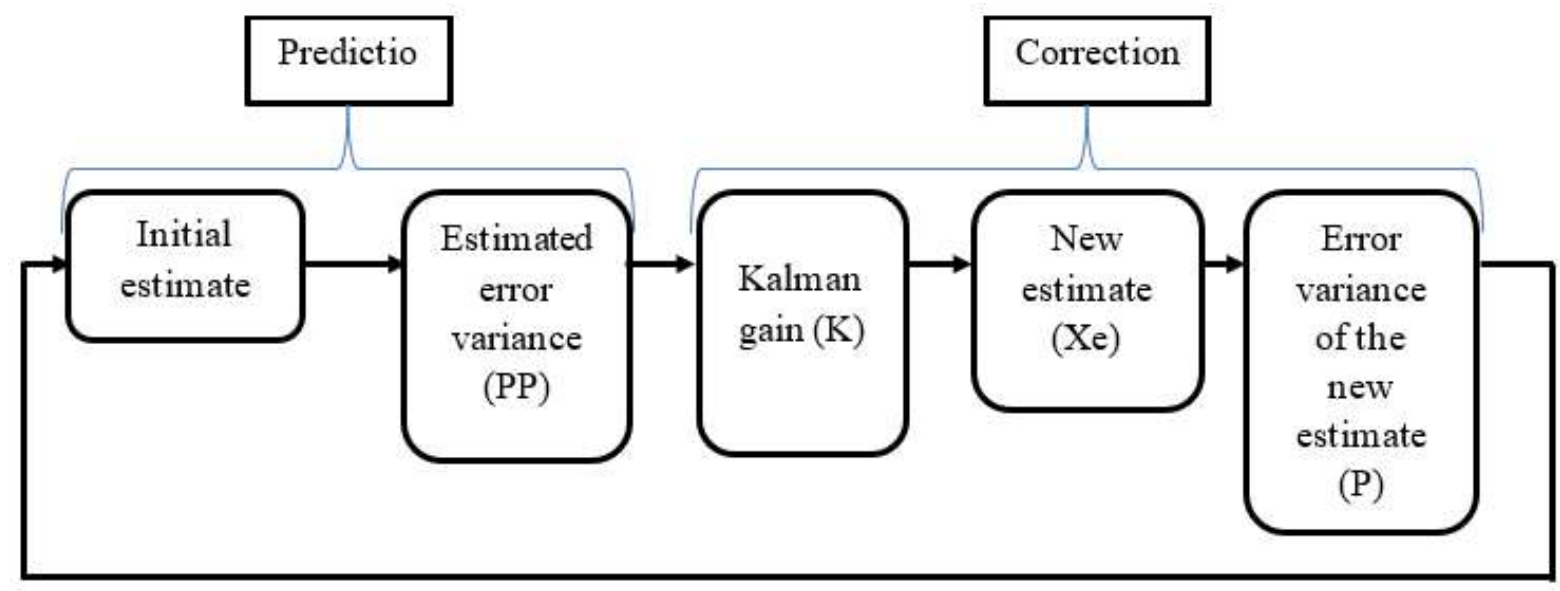

FIGURE 2. Stages of Kalman filtering.

The iterative process of Kalman filtering between the time and measurement updates started after initializing the GNSS position generation, as detailed by Xiong et al. (2014) with modifications. The system was propagated to the next time interval to obtain an estimate for the next position. In the $i+1$ position, the current state vector was updated to $\mathrm{Xi}_{\mathrm{i}+1}$. The first collected data were used as the first initial estimate (Xi) whereas the other data were calculated from [eq. (1)], as established in the first step. The variance of the initial estimate PP was calculated in the second step (equation 2). The GNSS maximum error, defined as $10 \mathrm{~m}$ by the manufacturer, was used as the perturbation and sensor variance parameter. 


$$
X i=a X e_{i}
$$

Where,

$\mathrm{Xi}$ is the initial estimate;

$\mathrm{a}$ is a constant of the linear system,

$\mathrm{Xe}_{\mathrm{i}}$ is the estimate at position $\mathrm{i}$.

$$
P P=P a^{2} W
$$

Where,

PP is the variance of the initial estimate;

$\mathrm{P}$ is the system variance,

$\mathrm{W}$ is the variance of the disturbance of the system.

For the last three stages, the noise in the measurement of the sensors that compose the system was evaluated, allowing obtaining a corrected measure of the system. In the third of the five steps, the Kalman gain (equation 3) was calculated and was used to find the new estimate $\left(\mathrm{Xe}_{\mathrm{i}+1}\right)$ in the fourth step (equation 4). This new estimate was the filter output, i.e., the estimate of the new point.

$$
K=\frac{M P P}{\left(P P M^{2}\right)+U}
$$

Where,

$\mathrm{K}$ is the Kalman gain;

$\mathrm{M}$ is the constant of the correction system,

$\mathrm{U}$ is the variance of the sensor measurement.

$$
X e_{i+1}=X i+y_{i+1}-(M X i)
$$

Where,

$\mathrm{Xe}_{\mathrm{i}+1}$ is the estimate at position $\mathrm{i}+1$,

$\mathrm{y}_{\mathrm{i}+1}$ is the noisy measurement at position $\mathrm{i}+1$ (filter input and, in the present study, position measurements).

In the fifth step, a new variance was calculated for the error of the new point (equation 5). The gain matrix was optimized to produce minimum error variance. The variance of the new point was used to calculate the next initial estimate, restarting the filter sequence.

$$
P_{i+1}=P P(1-K M)^{2}+U K^{2}
$$

Where,

$$
\mathrm{P}_{\mathrm{i}+1} \text { is the estimate variance at position } \mathrm{i}+1 .
$$

Every second, the sampled data were stored in a text file and displayed to the user using a graphical interface. The graphical interface was developed using the Qt Creator version 4.0.2 integrated development environment. An LCD touchscreen display (USD 75.00) was coupled to the BBB for data visualization and user interaction. The total cost of the GNSS-BBB system was USD 168.00.

\section{Data collection}

The experiments were conducted on October 15 and 16,2016 , on the campus of the Federal University of Viçosa (Universidade Federal de Viçosa [UFV], near the coordinates UTM $721399.29 \mathrm{~m} \mathrm{E}, 7701645.99 \mathrm{~m} \mathrm{~N}$, zone
23 S), Viçosa, Minas Gerais, Brazil, from 2:00 p.m. to 4:00 p.m. The position dilution of precision (PDOP) value of the collected points was set to less than 2.0 as a parameter of reliability. The PDOP value can be used to evaluate the performance of satellite geometry (Han et al., 2014), and minimum PDOP values suggest better satellite geometry and, therefore, more reliable data.

Three experiments were performed to analyze the GNSS-BBB integrated system: one collection of static data and two collections of kinematic data. The objective of this approach was to determine the accuracy error of the static and kinematic positioning points and system characteristics at different speeds. The data obtained with the proposed GNSS-BBB integrated system were transformed into UTM by the algorithm and stored in the platform for later analysis.

The static experiment consisted of demarcating a regular square grid containing the reference points. A regular square grid $(5 \times 5 \mathrm{~m})$ was defined in an area of 100.0 $\mathrm{m}^{2}$ with points every $2.5 \mathrm{~m}$. For this purpose, a topographic GNSS with a simple L1 frequency (model ProXT; Trimble Navigation Limited, Trimble, USA) that allows postprocessed differential correction was used. Data correction was performed using the GPS Pathfinder Office ${ }^{\circledR}$ software version 5.00 using data from the Brazilian Continuous Monitoring Network (Rede Brasileira de Monitoramento Continuo) of the Brazilian Institute of Geography and Statistics (Instituto Brasileiro de Geografia e Estatística). After correcting the reference data, the topographic GNSS indicated a mean error of $0.15 \mathrm{~m}$. In this same grid, 25 experimental points were randomly collected three times using the GNSS-BBB system proposed in this study.

In the first kinematic experiment, two parallel lines of $100.0 \mathrm{~m}$ were used. The objective of this experiment was to evaluate the behavior of the GNSS-BBB system before and after Kalman filtering as a function of velocity. For this purpose, four mean speeds were used: $1.39,2.22,2.78$, and $4.17 \mathrm{~m} \mathrm{~s}^{-1}$. The experiment was performed in triplicate considering the velocities in ascending order, and the mean values were considered for analysis.

In the second kinematic experiment, two tests were performed, with three repetitions each, at a mean velocity of $1.39 \mathrm{~m} \mathrm{~s}^{-1}$ in a random order. Four parallel lines of 100.0 $\mathrm{m}$ were used. The objective of this experiment was to determine system accuracy and the radius of the curvature required for the Kalman filter not to consider changes in direction as system errors. This is because Kalman filtering smoothes abrupt changes in direction, and some cycles of data collection are required for stabilization. To assess the distance required to change the direction of the Kalman filter, the four lines were followed by changing direction in the normal position, stretching the head to extend the curve, and evaluating the behavior of the filter.

\section{Measurements}

The static positioning errors $\left(\mathrm{PE}_{\mathrm{s}}\right)$ were calculated as the difference between the coordinates of the experimental points obtained with the GNSS-BBB system and the reference points obtained with the topographic GNSS. The errors in the data collected in the static experiment were determined using [eq. (6)]. 


$$
P E_{S}=\sqrt[2]{E N^{2}+E L^{2}}
$$

Where,

$\mathrm{PE}_{\mathrm{s}}$ is the static position error;

EN is the error in the north direction in meters,

$\mathrm{EL}$ is the error in the east direction in meters.

The kinematic position errors $\left(\mathrm{PE}_{\mathrm{k}}\right)$ were calculated according to [eq. (7)]. This determines the distance between the coordinates of the experimental points $\left(\mathrm{x}_{\mathrm{i}}, \mathrm{y}_{\mathrm{i}}\right)$ obtained with the GNSS-BBB system and the straight line $(a x+b y+c=0)$ formed by the reference points obtained with the topographic GNSS. The means of the errors between the GNSS-BBB system before and after filtering were compared using Student's $t$-test at a level of significance of $5 \%$.

$$
E P_{k}=\frac{\left|a x_{i}+b y_{i}+c\right|}{\sqrt[2]{a^{2}+b^{2}}}
$$

Where,

Ep $\mathrm{p}_{\mathrm{k}}$ is the kinematic positioning error;

$\mathrm{a}$ is the angular coefficient of the line on the $\mathrm{x}$-axis;

$\mathrm{x}_{\mathrm{i}}$ is the east UTM coordinate;

$\mathrm{b}$ is the angular coefficient on the y-axis;

$\mathrm{y}_{\mathrm{i}}$ is the north UTM coordinate;

$\mathrm{c}$ is the distance from the origin $(0,0)$;

$\mathrm{i}$ is the number of points collected

The root-mean square error (RMSE) (equation 8), which indicates data accuracy, does not use the mean values and produces an absolute error relative to the reference value, representing $68 \%$ of the distribution (Machado \& Molin, 2011).

$$
R M S E=\sqrt{\frac{\sum_{i=1}^{n} E P^{2}}{n}}
$$

Where,

RMSE is the root-mean square error;

$\mathrm{PE}$ is the static or kinematic position error, $\mathrm{n}$ is the number of points collected.

\section{RESULTS AND DISCUSSION}

\section{Analysis of the GNSS-BBB system in the static experiment}

The position errors varied from 0.79 to $6.26 \mathrm{~m}$ (Figure 3) with a mean error of $3.35 \mathrm{~m}$ and RMSE of 3.73 $\mathrm{m}$ relative to the position obtained using the topographic GNSS. The results of the static experiment corroborate those found by Machado \& Molin (2011), who analyzed eight GNSS receivers and found that uncorrected RMSE values varied from 1.02 to $3.11 \mathrm{~m}$.

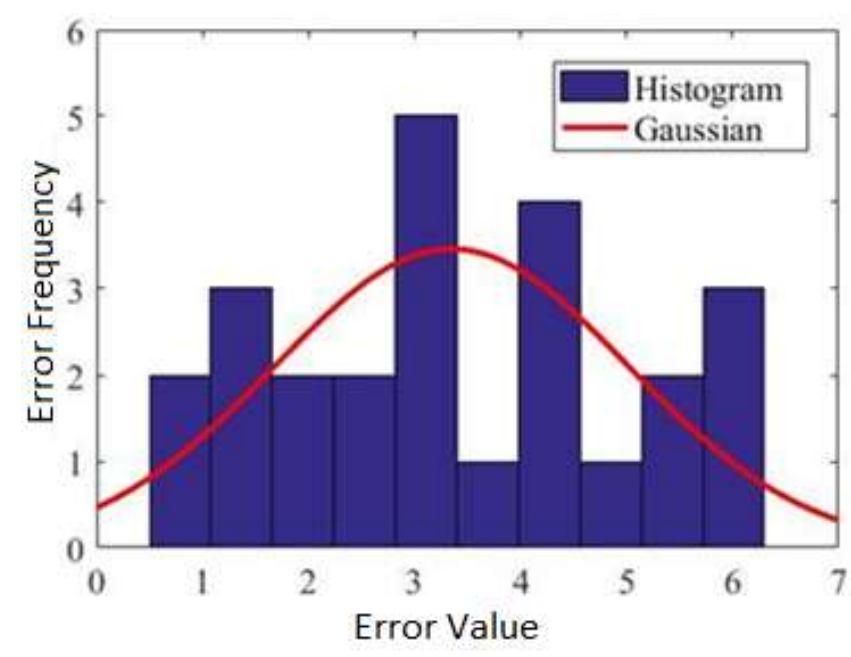

FIGURE 3. Frequency error between the topographic GNSS and the GNSS module integrated to the BeagleBoard Black for the static experiment

There was no tendency of decrease in the errors as a function of the increase in data collection time because the GNSS position data remained constant and were modified only if the system was in motion.

\section{Analysis of the GNSS-BBB system in the first kinematic experiment}

The data collected at different speeds are shown in Figure 4. The UTM coordinates were deduced from 721200 $\mathrm{m}$ in the east to west direction and $7701500 \mathrm{~m}$ in the north to south direction to obtain lower coordinates. The coordinates can be returned to the original values by adding these values to the coordinates shown in the graph. Among the four mean velocities evaluated, the velocity of $1.39 \mathrm{~m} \mathrm{~s}^{-1}$ presented lower lateral oscillation and lower mean error and RMSE (Table 1). In addition, the mean velocity increased as the data presented greater lateral oscillations and, consequently, higher mean errors and RMSE. 
A.

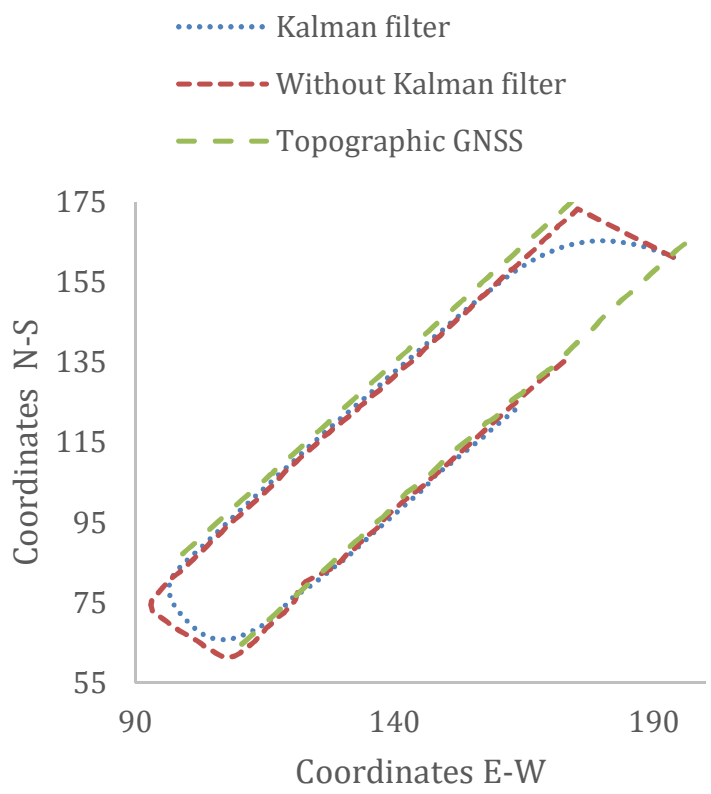

C.

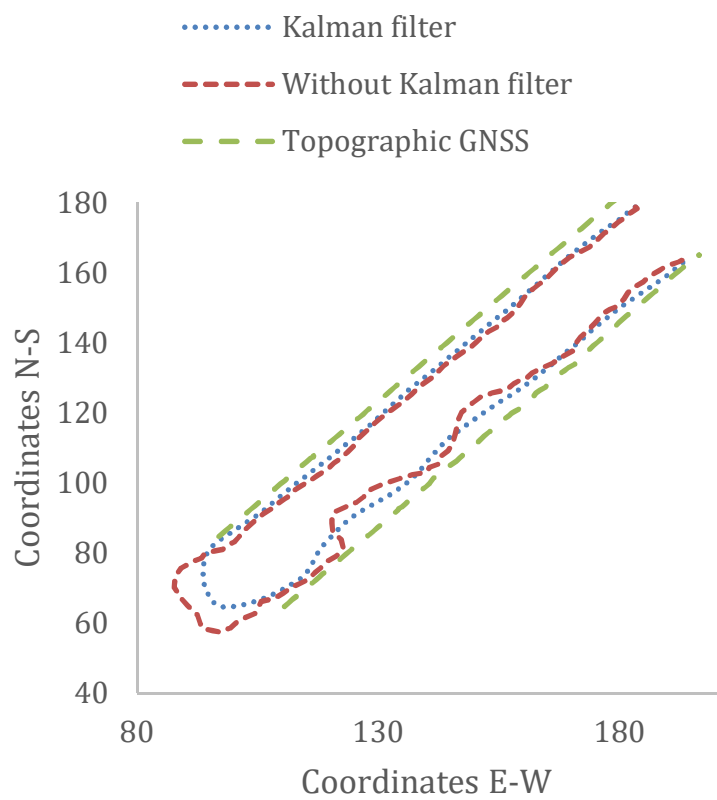

B.
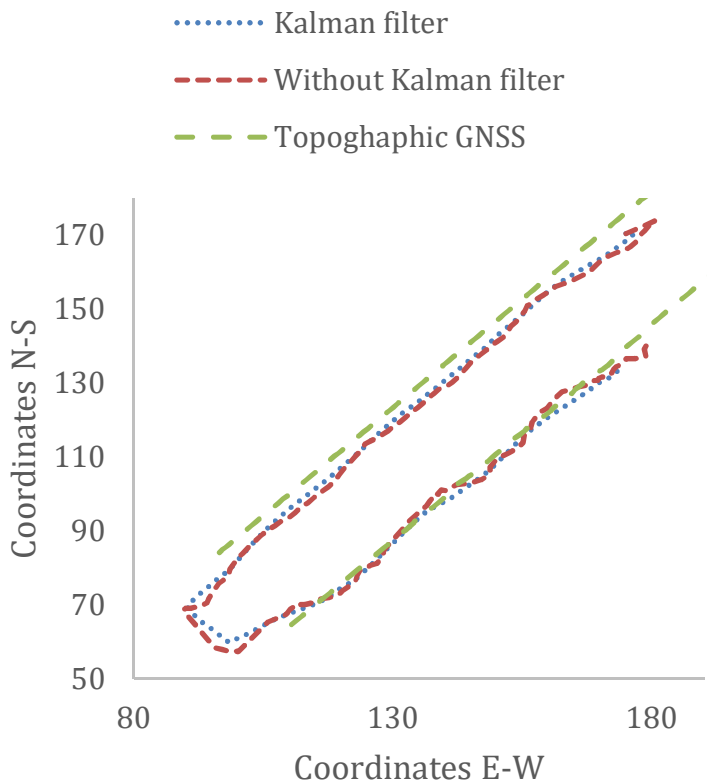

D.
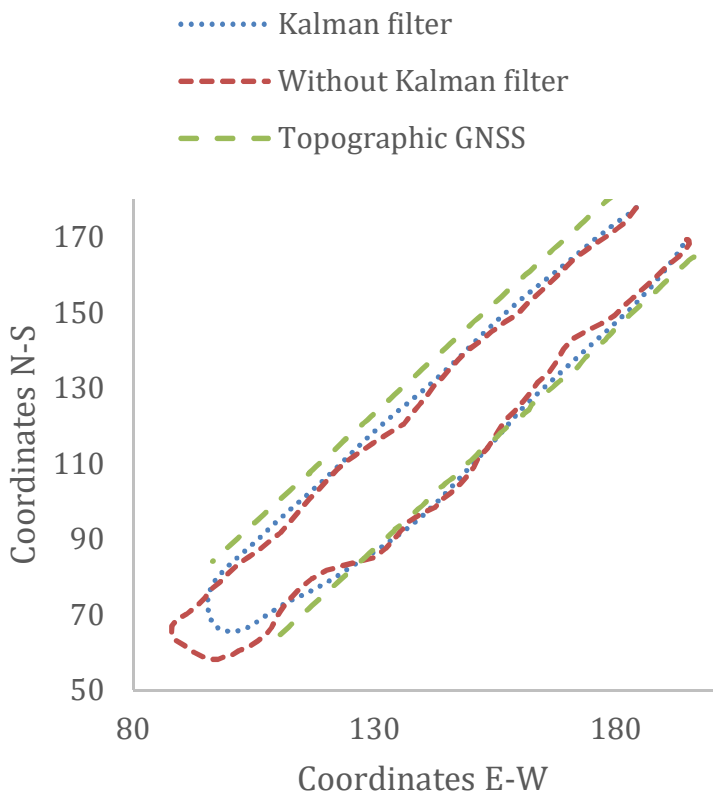

FIGURE 4. Trajectories demarcated by the GNSS-BBB system at mean speeds of $1.39 \mathrm{~m} \mathrm{~s}^{-1}(\mathrm{~A}), 2.22 \mathrm{~m} \mathrm{~s}^{-1}$ (B), $2.78 \mathrm{~m} \mathrm{~s}^{-1}$ (C) and $4,17 \mathrm{~m} \mathrm{~s}^{-1}(\mathrm{D})$.

TABLE 1. Descriptive statistics of the errors obtained in the kinematic experiment at four different mean speeds with and without Kalman filtering.

\begin{tabular}{ccccccc}
\hline \multirow{2}{*}{ Speed $(\mathrm{m} / \mathrm{s})$} & \multirow{2}{*}{ Kalman filtering } & \multicolumn{4}{c}{ Errors $(\mathrm{m})$} & \multirow{2}{*}{ SD } \\
\cline { 3 - 6 } & & Minimum & Maximum & Mean & RMSE & \\
\hline \multirow{2}{*}{1.39} & $\mathrm{P}$ & 0.01 & 2.62 & 1.27 & 1.34 & 0.40 \\
& $\mathrm{~A}$ & 0.07 & 2.67 & 1.50 & 1.52 & 0.20 \\
2.22 & $\mathrm{P}$ & 0.01 & 5.03 & 2.37 & 2.57 & 0.91 \\
& $\mathrm{~A}$ & 0.09 & 7.04 & 2.73 & 2.92 & 1.00 \\
2.78 & $\mathrm{P}$ & 0.72 & 5.99 & 2.96 & 3.22 & 1.24 \\
& $\mathrm{~A}$ & 1.12 & 9.56 & 3.59 & 4.00 & 1.51 \\
4.17 & $\mathrm{P}$ & 0.09 & 5.92 & 3.32 & 3.56 & 1.19 \\
& $\mathrm{~A}$ & 0.15 & 6.64 & 4.25 & 4.39 & 1.00 \\
\hline
\end{tabular}

${ }^{\mathrm{P} D a t a}$ obtained by the GNSS-BBB integrated system with Kalman filtering; ${ }^{\mathrm{A}}$ Data obtained by the integrated GNSS-BBB system without Kalman filtering; RMSE, root-mean square error; SD, standard deviation. 
Kalman filtering reduced the mean errors, RMSE, and lateral oscillations in all cases. The decrease in lateral oscillations is fundamental for implementing automatic navigation in agricultural machinery so that the system does not undergo sudden changes in direction. Data linearity enables this GNSS-BBB system to be used in the automatic navigation of agricultural operations where submetric accuracy is not required, including soil sampling and crop yield monitoring.

The mean error, RMSE, and standard deviation tended to increase as velocity increased (Table 1). The data tended to present smaller minimum, maximum, mean, and RMSE errors using Kalman filtering. The standard deviations were higher with Kalman filtering at speeds of 1.39 and $4.17 \mathrm{~m} \mathrm{~s}^{-1}$. This result was probably due to the absence of gross and abrupt errors at these speeds (Figure 4).

A.
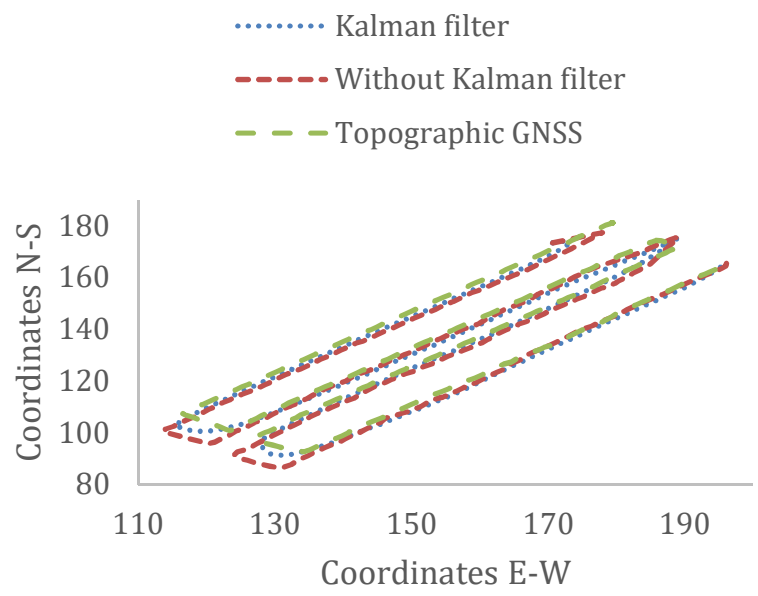

Analysis of the GNSS-BBB system in the second kinematic experiment

The distance required to change the direction of the Kalman filter was simulated in two tests by changing the direction of the normal position (Figure 5a) and lengthening the head to extend the curve (Figure 5b). The UTM coordinates were deduced from $721200 \mathrm{~m}$ in the east to west direction and $7701500 \mathrm{~m}$ in the north to south direction to obtain lower coordinates. The coordinates can be returned to the original values by adding these values to the coordinates shown in the graph. The reference data collected with the topographic GNSS were compared with data collected with the proposed system before and after Kalman filtering. The data before filtering presented oscillations, although the trajectory was linear. The data after filtering presented smaller deviations and were closer to the topographic GNSS data.

B.

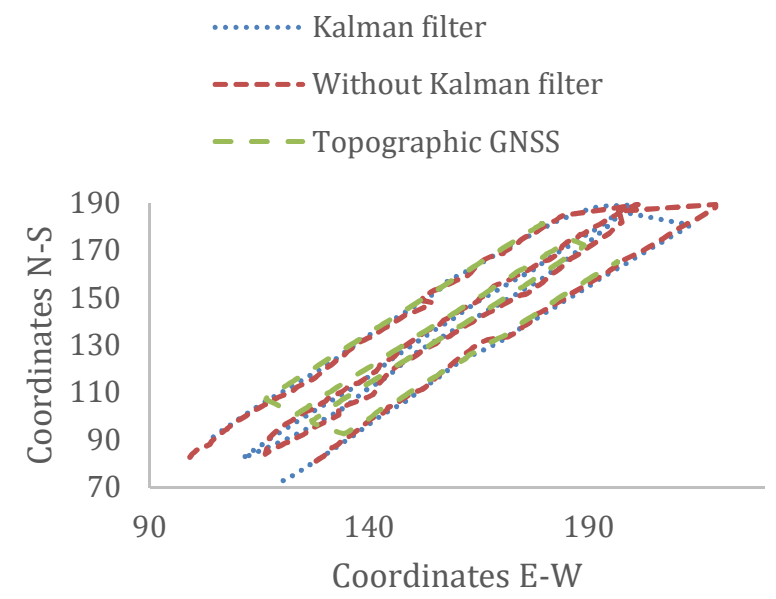

FIGURE 5. Kinematic experiment with a change in the direction of the normal position (A) and lengthening the head to extend the curve (B).

The mean position errors were 1.26 and $1.13 \mathrm{~m}$, and RMSE was 1.45 and $1.27 \mathrm{~m}$ for the data obtained before and after Kalman filtering, respectively, relative to the topographic GNSS. The results of Student's $t$-test indicated that after Kalman filtering, the mean error of the data was significantly lower than the error obtained without filtering $(p<0.05)$. The improvement is because Kalman filtering maintains the system characteristics, smoothing abrupt changes and consequently decreasing positioning errors (Gil et al., 2013). Kalman filtering removes from the signal a large part of random errors, decreasing the maximum error of the data from 3.58 to $2.82 \mathrm{~m}$ after filtering, corroborating the results of Gil et al. (2013) and Han et al. (1998).

However, considering that the Kalman filter is not indicated for curved trajectories (Han et al., 1998) because it maintains the tendency of the data and rejects the data obtained from a change in direction. At the speed of $1.39 \mathrm{~m}$ $\mathrm{s}-1$, it took approximately $5 \mathrm{~m}$ for the Kalman filter to follow the data obtained from a correct change in direction.

\section{CONCLUSIONS}

Kalman filtering increased the accuracy of kinematic data compared to static data by eliminating sudden lateral oscillations due to random errors of the GNSS signal, maintaining a linear pattern, and decreasing the errors in all cases.

The GNSS module integrated into the BBB can be used in specific agricultural applications where submetric accuracy is not required, including soil sampling and productivity monitoring. These operations should be performed at a speed of $1.39 \mathrm{~m} \mathrm{~s}^{-1}$ and a minimum radius of curvature greater than $5 \mathrm{~m}$ because Kalman filtering did not produce good results in the sections that changed direction.

\section{ACKNOWLEDGMENTS}

The authors are grateful to $\mathrm{CNPq}, \mathrm{CAPES}$, and FAPEMIG for funding this research. 


\section{REFERENCES}

Bakker T, Van Asselt K, Bontsema J, Mülher J, Straten GV (2011) Autonomous navigation using a robot platform in a sugar beet field. Biosystems Engineering 109(1):357368. DOI: https://doi.org/10.1016/j.biosystemseng.2011.05.001

BeagleBoard Black (2019) Beaglebone black. In: What is Beaglebone black? Available:

https://beagleboard.org/black. Accessed: Mar 13, 2019.

Chen J, Shi SB, Hou JC, Xu J (2005) The Development of Digital Agriculture and Chinese Agriculture Machinery. Journal of Agricultural Mechanization Research 3:008.

Cintra RS, Velho HFC, Todling R (2010) Redes neurais artificiais na melhoria de desempenho de métodos de assimilação de dados: Filtro de Kalman. Trends in Applied and Computational Mathematics 11:29-39. DOI: https://doi.org/10.5540/tema.2010.011.01.0029

De Oliveira RP (2016) Apoio à Decisão na Adoção da Agricultura de Precisão: A Tecnologia da Informação em Apoio ao Conhecimento Agronômico. Revista Eletrônica Competências Digitais para Agricultura Familiar 2:89-109.

Dutch S (2015) Converting UTM to Latitude and Longitude (Or Vice Versa). Available:

http://www.uwgb.edu/dutchs/UsefulData/UTMFormulas.H TM. Accessed Mar 14, 2017.

Fourati H (2015) Heterogeneous data fusion algorithm for pedestrian navigation via foot-mounted inertial measurement unit and complementary filter. IEEE Transactions on Instrumentation and Measurement 64(1):221-229. DOI: https://doi.org/10.1109/TIM.2014.2335912

Gil JG, Gonzalez RR, Garcia SA, Gil FJG (2013) A kalman filter implementation for precision improvement in low-cost GPS positioning of tractors. Sensors 13:1530715323. DOI: https://doi.org/10.3390/s131115307

Han S, Gui Q, Li G, Du Y (2014) Minimum of PDOP and its applications in inter-satellite links (ISL) establishment of Walker- $\delta$ constellation. Advances in Space Research 54:726-733. DOI: https://doi.org/10.1016/j.asr.2014.04.020

Han S, Zhang Q, Noh HK (1998) Applying filtering techniques to improve GPS positioning accuracy. In: 2001 ASAE Annual Meeting. American Society of Agricultural and Biological Engineers. CD.

Huang Y, Zhang Y, Xu B, Wu Z, Chambers JA (2018) A new adaptive extended Kalman filter for cooperative localization. IEEE Transactions on Aerospace and Electronic Systems 54(1):353-368. DOI: https://doi.org/10.1109/TAES.2017.2756763

Ji W, Zhao D, Cheng F, Xu B, Zhang Y, Wang J (2012) Automatic recognition vision system guided for apple harvesting robot. Computers and Electrical Engineering 38(1):1186-1195. DOI:

https://doi.org/10.1016/j.compeleceng.2011.11.005
Kalman RE (1960) A new approach to linear filtering and prediction problems. Journal of basic Engineering 82:3545. DOI: https://doi.org/10.1115/1.3662552

Khan A, Mishra R (2012) GPS-GSM based tracking system. International Journal of Engineering Trends and Technology 3:161-164.

Laveti G, Rao GS, Bidikar B (2016) Modified Kalman Filter for GPS Position Estimation over the Indian Sub Continent. Procedia Computer Science 87:198-203. DOI: https://doi.org/10.1016/j.procs.2016.05.148

Li J, Song N, Yang G, Li M, Cai Q (2017) Improving positioning accuracy of vehicular navigation system during GPS outages utilizing ensemble learning algorithm. Information Fusion 35:1-10. DOI: https://doi.org/10.1016/j.inffus.2016.08.001

Li K, Chang L, Hu B (2016) A Variational BayesianBased Unscented Kalman Filter With Both Adaptivity and Robustness. IEEE Sensors Journal 16:6966-6976. DOI: https://doi.org/10.1109/jsen.2016.2591260

Liu Y, Fan X, Lv C, Wu J, Li L, Ding D (2018) An innovative information fusion method with adaptive Kalman filter for integrated INS/GPS navigation of autonomous vehicles. Mechanical Systems and Signal Processing 100:605-616. DOI:

https://doi.org/10.1016/j.ymssp.2017.07.051

Machado TM, Molin JP (2011) Ensaios estáticos e cinemáticos de receptores de GPS. Revista Brasileira de Engenharia Agrícola e Ambiental 15:981-988. DOI: https://doi.org/10.1590/s1415-43662011000900015

Mondal P, Basu M, Bhadoria PBS (2011) Critical review of precision agriculture technologies and its scope of adoption in India. American Journal of Experimental Agriculture 1:49-68. DOI:

https://doi.org/10.9734/AJEA/2011/155

Mousazadeh H (2013) A technical review on navigation systems of agricultural autonomous off-road vehicles. Journal of Terramechanics 50(3):211-232. DOI: DOI: https://doi.org/10.1016/j.jterra.2013.03.004

Olesen D, Jakobsen J, Benzon HH, Knudsen P (2016) GNSS Software Receiver for UAVs. European Journal of Navigation.

Shen JC, Chang CH, Wu SJ, Hsu CT, Lien HC (2015) Real-time correction of water stage forecast using combination of forecasted errors by time series models and Kalman filter method. Stochastic environmental research and risk assessment 29(7):1903-1920. DOI: https://doi.org/10.1007/s00477-015-1074-9 
Suprem A, Mahalik N, Kim K (2013) A review on application of technology systems, standards and interfaces for agriculture and food sector. Computer Standards \& Interfaces 35:355-364. DOI: https://doi.org/10.1016/j.csi.2012.09.002

Xiong B, Zhao J, Wei Z, Skyllas-Kazacos M (2014) Extended Kalman filter method for state of charge estimation of vanadium redox flow battery using thermaldependent electrical model. Journal of Power Sources 262:50-61. DOI: https://doi.org/10.1016/j.jpowsour.2014.03.110
Xiong H, Tang J, Xu H, Zhang W, Du Z (2018) A Robust Single GPS Navigation and Positioning Algorithm Based on Strong Tracking Filtering. IEEE Sensors Journal 18(1):290-298. DOI: https://doi.org/10.1109/jsen.2017.2767066

Xu G, Xu Y (2016) GPS: theory, algorithms and applications. New York, Springer, 3ed. 488p.

Yin, X, Noguchi N (2013) Development of a target following system for a field robot. IFAC Proceedings Volumes 46(18):109-114. DOI:

https://doi.org/10.3182/20130828-2-SF-3019.00068 